\title{
Quadruplex-based Molecular Beacons as Tunable DNA Probes
}

\author{
A. Bourdoncle, ${ }^{1,2}$ A. Estévez Torres,${ }^{1}$ C. Gosse,${ }^{3, *}$ L. Lacroix,${ }^{2}$ \\ P. Vekhoff, ${ }^{2}$ T. Le Saux, ${ }^{1}$ L. Jullien, ${ }^{1, *}$ J.-L. Mergny ${ }^{2, *}$
}

1) Département de Chimie, CNRS UMR 8640, Ecole Normale Supérieure, 24 rue Lhomond 75005, Paris Cedex 05, France; 2) Laboratoire de Biophysique, Muséum National d'Histoire USM503, INSERM U 565, CNRS UMR 5153, 43 rue Cuvier, 75005, Paris Cedex 05, France; 3) Laboratoire de Photonique et de Nanostructures, LPN-CNRS, Route de Nozay, 91460 Marcoussis, France

June 22, 2006

\section{Supporting Information}

- Supplementary Materials

- Supplementary Figures

* Change in the absorbance (Figure 1Sa) and in the ellipticity (Figure 1Sb) with respect to temperature of $30 \mathrm{G}$ solutions in $100 \mathrm{mM} \mathrm{KCl} 10 \mathrm{mM} \mathrm{MgCl}$ $10 \mathrm{mM}$ lithium cacodylate $\mathrm{pH}$ 7.2,

* Change in the absorbance at $295 \mathrm{~nm}$ with respect to temperature of $30 \mathrm{G}$ solutions at different concentrations in $100 \mathrm{mM} \mathrm{KCl} 10 \mathrm{mM}$ lithium cacodylate pH 7.2 (Figure $2 \mathrm{~S}$ ),

* Influence of the loop length on the enthalpic and entropic contributions to the Gibbs free energy associated to the formation of the quadruplex motif (Figure 3S),

* Temperature dependence of the 30GMB fluorescence emission or of the $30 \mathrm{G}$ absorbance in the presence of $13 \mathrm{ACA}, 13 \mathrm{ACC}$, or $13 \mathrm{AAA}$ in $100 \mathrm{mM}$ $\mathrm{KCl} 10 \mathrm{mM} \mathrm{MgCl} 210 \mathrm{mM}$ lithium cacodylate buffer $\mathrm{pH} 7.2$ (Figure 4S),

* Free energy relationships $\Delta_{21} \mathrm{H}^{\circ}\left(\Delta_{21} \mathrm{~S}^{\circ}\right)$ for G4-MB and traditional molecular beacons (Figure 12S).

- Supplementary Tables. 
* Thermodynamic data associated to the intramolecular formation of a three quartet quadruplex motif for the nonlabeled oligonucleotide sequences (Table $1 \mathrm{~S})$.

- Appendices:

- Appendix A: Evaluation of the relevance of the thermodynamic parameters extracted from analysis of thermal curves with a two-level model when more than one closed G4-MB is formed;

- Appendix B: Detailed analysis of the kinetics of association between 30GMB and its complementary targets;

- Appendix C: Electrophoretic measurements. 


\section{Supplementary Materials}

\section{Supplementary Figures}

a)

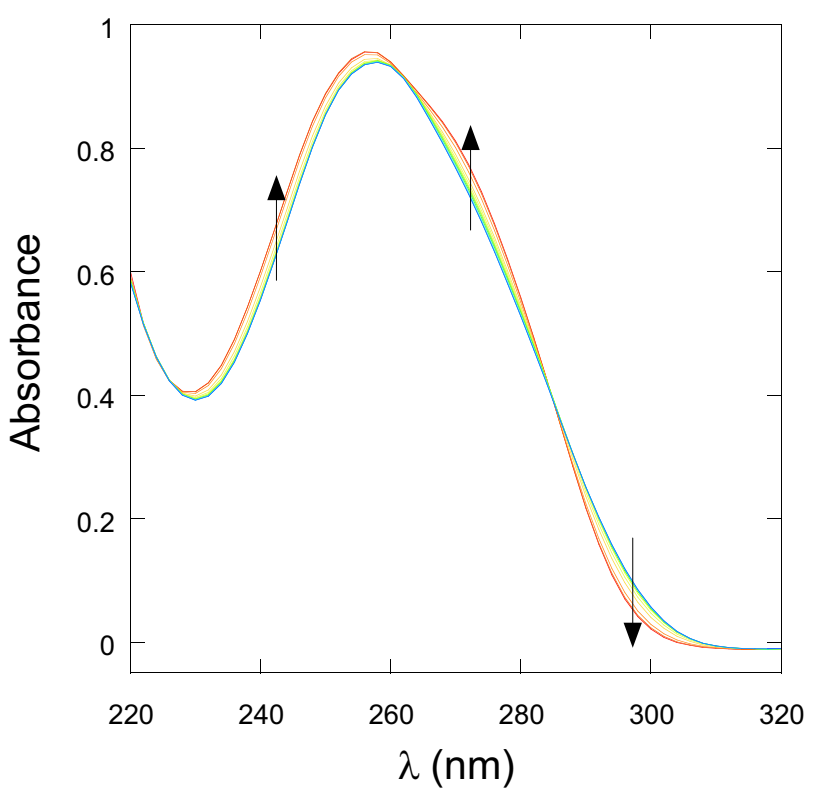

b)

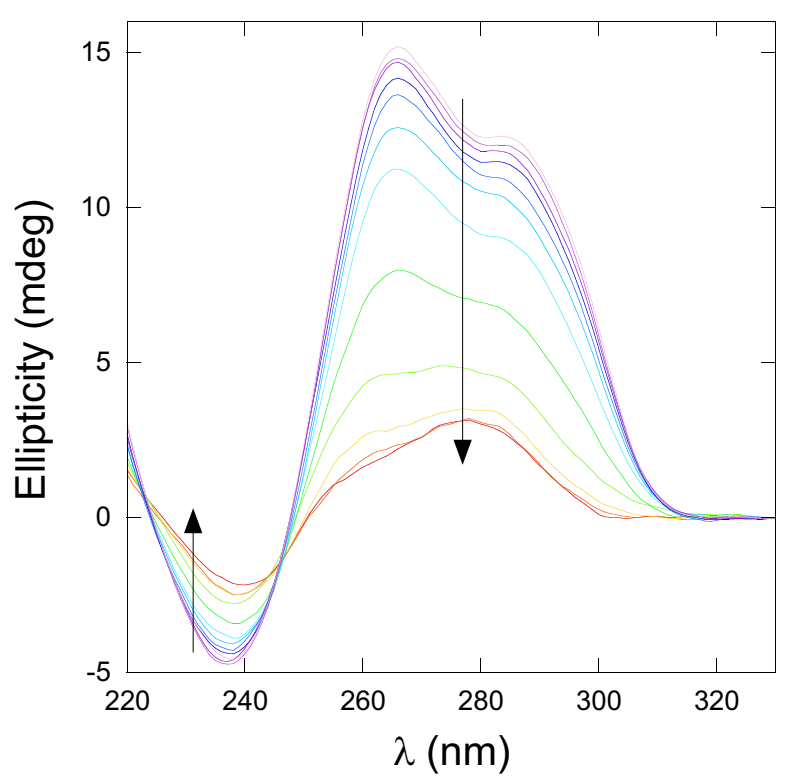

Figure 1S. Change in the absorbance (a) and in the ellipticity (b) upon increasing temperature (from blue to red) of $30 \mathrm{G}$ solutions in $100 \mathrm{mM} \mathrm{KCl} 10 \mathrm{mM} \mathrm{MgCl} 210 \mathrm{mM}$ lithium cacodylate $\mathrm{pH}$ 7.2. a: $3 \mu \mathrm{M}$ in a cuvette with a $10 \mathrm{~mm}$ light path length, $\mathrm{T}\left({ }^{\circ} \mathrm{C}\right)=75.0,69.8,64.6,59.2,55.6$, $50.2,44.8,39.4,34.1,30.6,25.3,23.9 ; \mathbf{b}: 15 \mu \mathrm{M}$ in a cuvette with a $2 \mathrm{~mm}$ light path length, $\mathrm{T}\left({ }^{\circ} \mathrm{C}\right)=84,80,75,70,65,60,55,50,45,40,35,25$. At low temperature, the spectrum compares well with reported results for G4 [1]. Despite the solvent expansion with the temperature change, one notices the presence of sharp isosbestic $(1 \mathrm{Sa})$ or isoelliptic $(1 \mathrm{Sb})$ points in both Figures. 


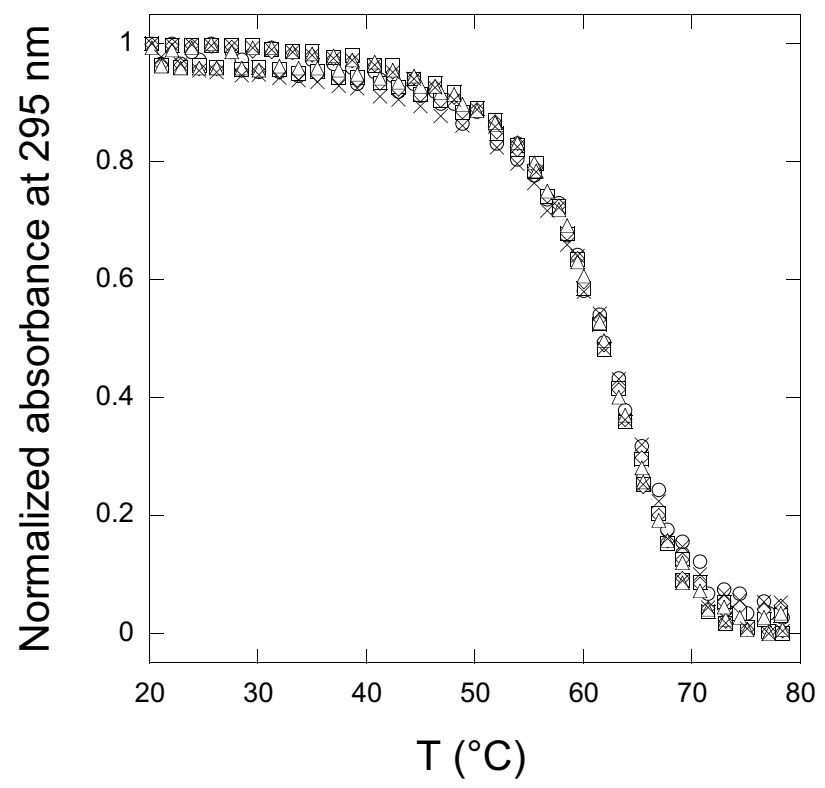

Figure 2S. Change in the absorbance at $295 \mathrm{~nm}$ with respect to temperature of $30 \mathrm{G}$ solutions at different strand concentrations in $100 \mathrm{mM} \mathrm{KCl} 10 \mathrm{mM}$ lithium cacodylate $\mathrm{pH}$ 7.2. $30 \mathrm{G}$ concentrations: 1 (circles), 2 (squares), 5 (diamonds), 10 (triangles) and 25 (crosses) $\mu \mathrm{M}$. Maximum and minimum values were normalized to 1 and 0 for comparison. 


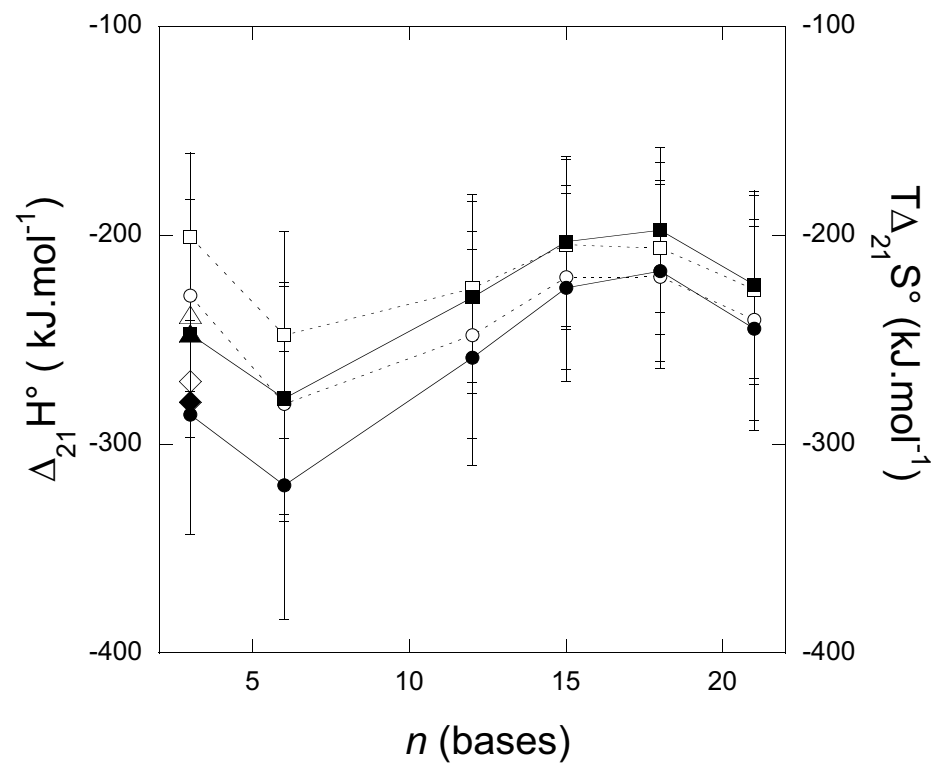

Figure 3S. Influence of the loop length on the enthalpic $\Delta_{21} H^{0}$ (circles) and entropic $T \Delta_{21} S^{0}$ (squares) contributions to the Gibbs free energy $\Delta_{21} G^{0}$ associated to the formation of the quadruplex motif in the presence (filled markers) or in the absence (empty markers) of $10 \mathrm{mM} \mathrm{MgCl}_{2}$ at $298 \mathrm{~K}$. The corresponding data for the 21 tel sequence are displayed as diamonds for the enthalpic contribution and as triangles for the entropic contribution (filled markers: $10 \mathrm{mM} \mathrm{MgCl}_{2}$; empty markers: no $\mathrm{MgCl}_{2}$ ). 
a)

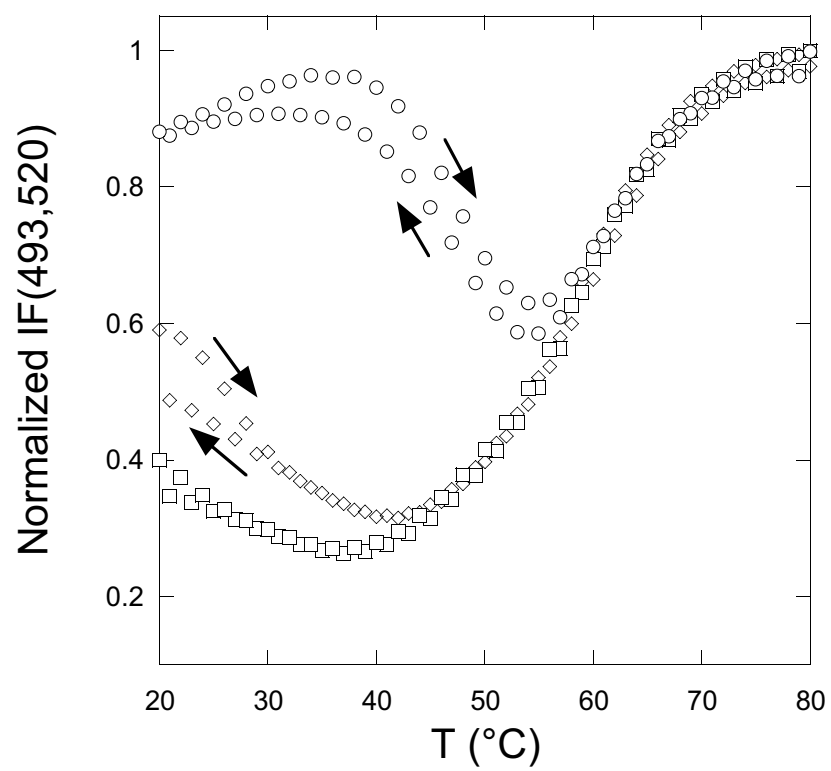

b)

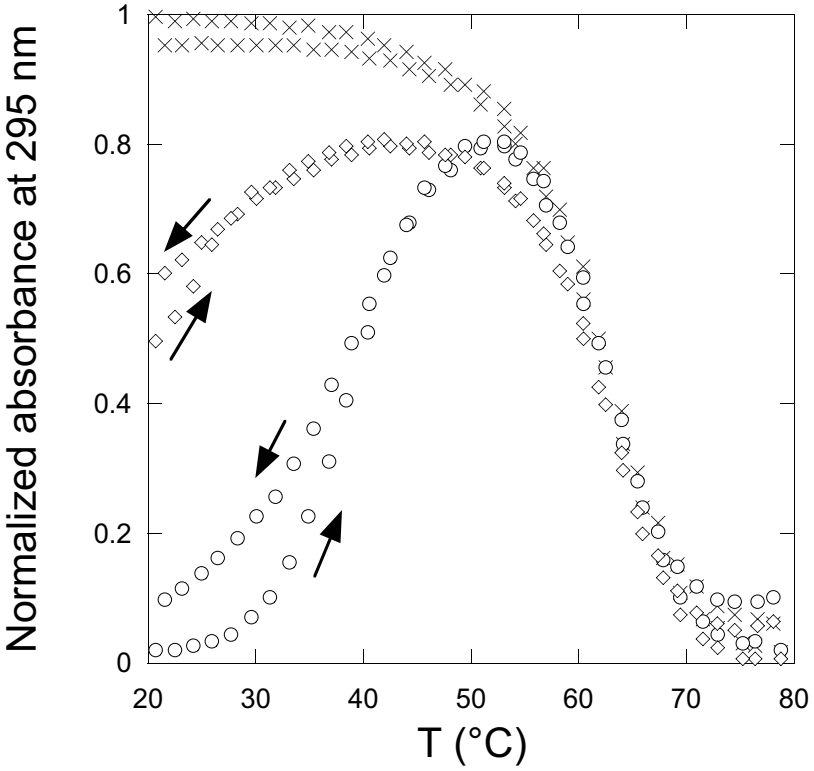

Figure 4S. a: Temperature dependence of the fluorescence emission (normalized by homothetic transformation at $80^{\circ} \mathrm{C}$ ) from $50 \mathrm{nM} 30 \mathrm{GMB}$ in the presence of $6 \mu \mathrm{M}$ in 13ACA (circles), 13ACC (diamonds), and 13AAA (squares). $\lambda_{e x c}=493 \mathrm{~nm} ; \lambda_{e m}=520 \mathrm{~nm}$; b: Temperature dependence of the absorbance at $295 \mathrm{~nm}$ (normalized at $80^{\circ} \mathrm{C}$ ) of $2 \mu \mathrm{M} 30 \mathrm{G}$ alone (crosses) or in the presence of 1 equivalent of 13ACA (circles) or 13ACC (diamonds). Solvent: $100 \mathrm{mM} \mathrm{KCl} 10 \mathrm{mM} \mathrm{MgCl} 2$ $10 \mathrm{mM}$ lithium cacodylate buffer $\mathrm{pH}$ 7.2. Both cooling and heating curves are shown; the arrows indicate the directions of the temperature change. 


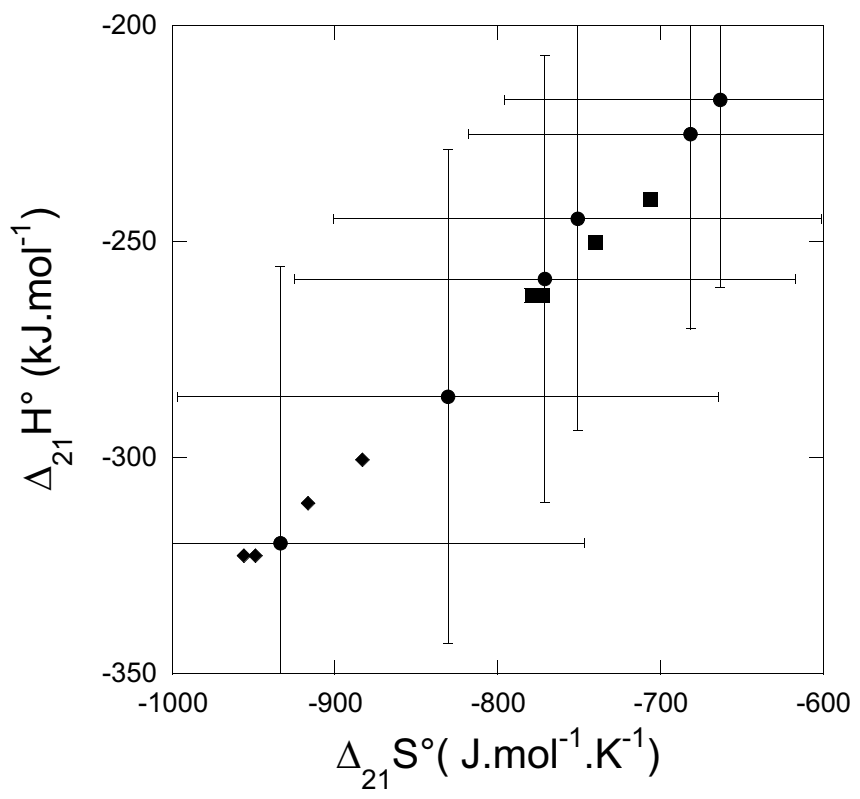

Figure 12S. Free energy relationships $\Delta_{21} \mathrm{H}^{\mathrm{o}}\left(\Delta_{21} \mathrm{~S}^{\mathrm{o}}\right)$ for G4-MB and traditional Watson-Crick molecular beacons. G4-MB data (disks) are extracted from Table 1S (solvent: $100 \mathrm{mM} \mathrm{KCl} 10$ $\mathrm{mM} \mathrm{MgCl}_{2} 10 \mathrm{mM}$ lithium cacodylate buffer $\mathrm{pH}$ 7.2). The corresponding data for the traditional molecular beacons were calculated by using the Mfold program [2] with $100 \mathrm{mM} \mathrm{Na}{ }^{+} 10$ $\mathrm{mM} \mathrm{Mg}^{2+}$. Two stems: GACATGAC-loop-GTCATGTC (squares) and GATCATGTAC-loopGTACATGATC (diamonds), and four loop sequences: TGT, TTTGTT, CTTTGTTT, and TCCTTTGTTTGT were analyzed. One should note that in order to generate traditional MB with comparable $T_{m}$ and $\left(\Delta_{21} \mathrm{H}^{\mathrm{o}}, \Delta_{21} \mathrm{~S}^{\mathrm{o}}\right)$ values in the range of the one of the G4-MB, relative long stem were required. In the case of more classical traditional MB with 5-7 bp stem and with a $T_{m}$ comparable to the one of the G4-MB, $\left(\Delta_{21} \mathrm{H}^{\mathrm{o}}, \Delta_{21} \mathrm{~S}^{\mathrm{o}}\right)$ values were lower in absolute value. 


\section{Supplementary Tables}

Table 1S. Thermodynamic data associated to the intramolecular formation of a three quartet quadruplex motif for the nonlabeled oligonucleotide sequences displayed in Table 1. Data associated to the human telomeric motif 21 tel are also displayed as references. Experimental conditions : $2 \mu \mathrm{M}$ oligonucleotide in $10 \mathrm{mM}$ lithium cacodylate buffer $\mathrm{pH} 7.2$ containing $100 \mathrm{mM}$ $\mathrm{KCl}$ in the presence or in the absence of $10 \mathrm{mM} \mathrm{MgCl}_{2}$.

\begin{tabular}{|c|c|c|c|c|c|}
\hline Sequence & $n$ & $\begin{array}{c}T_{m} \pm 1 \\
\left({ }^{\circ} \mathrm{C}\right) \\
\end{array}$ & $\begin{array}{c}\Delta_{21} H^{\mathrm{o}} \pm 20 \% \\
\left(\mathrm{~kJ} \cdot \mathrm{mol}^{-1}\right) \\
\end{array}$ & $\begin{array}{c}\Delta_{21} S^{\mathrm{o}} \pm 20 \% \\
\left(\mathrm{~J} . \mathrm{K}^{-1} \cdot \mathrm{mol}^{-1}\right) \\
\end{array}$ & $\begin{array}{c}\Delta_{21} G^{\mathrm{o}}(298 K) \pm 20 \% \\
\left(\mathrm{~kJ} \cdot \mathrm{mol}^{-1}\right)\end{array}$ \\
\hline $39 \mathrm{G}(\mathrm{KCl})$ & 21 & 44 & -240 & -760 & -14 \\
\hline $39 \mathrm{G}\left(\mathrm{KCl}, \mathrm{MgCl}_{2}\right)$ & 21 & 53.5 & -245 & -750 & -21 \\
\hline $36 \mathrm{G}(\mathrm{KCl})$ & 18 & 45 & -220 & -690 & -14 \\
\hline $36 \mathrm{G}\left(\mathrm{KCl}, \mathrm{MgCl}_{2}\right)$ & 18 & 55 & -215 & -665 & -20 \\
\hline $33 \mathrm{G}(\mathrm{KCl})$ & 15 & 48 & -220 & -685 & -13 \\
\hline $33 \mathrm{G}\left(\mathrm{KCl}, \mathrm{MgCl}_{2}\right)$ & 15 & 58 & -225 & -680 & -22 \\
\hline $30 \mathrm{G}(\mathrm{KCl})$ & 12 & 55 & -250 & -770 & -22 \\
\hline $30 \mathrm{G}\left(\mathrm{KCl}, \mathrm{MgCl}_{2}\right)$ & 12 & 63 & -260 & -760 & -29 \\
\hline $24 \mathrm{G}(\mathrm{KCl})$ & 6 & 65 & -280 & -830 & -33 \\
\hline $24 \mathrm{G}\left(\mathrm{KCl}, \mathrm{MgCl}_{2}\right)$ & 6 & 70 & -320 & -935 & -42 \\
\hline $21 \mathrm{G}(\mathrm{KCl})$ & 3 & 67 & -230 & -675 & -28 \\
\hline $21 \mathrm{G}\left(\mathrm{KCl}, \mathrm{MgCl}_{2}\right)$ & 3 & 72 & -285 & -830 & -38 \\
\hline 21tel (KCl) & 3 & 67 & -270 & -800 & -33 \\
\hline 21tel $\left(\mathrm{KCl}, \mathrm{MgCl}_{2}\right)$ & 3 & 67 & -280 & -830 & -35 \\
\hline
\end{tabular}




\section{Appendix A: Evaluation of the relevance of the thermo- dynamic parameters extracted from analysis of thermal curves with a two-level model when more than one closed G4-MB is formed}

In tandem with capillary electrophoresis, the kinetic experiments associated to 30GMB folding suggested that several conformers coexist in the folded state (see Figure 4, Main Text and Figure 9-11S). At the same time, we used a two-level model to extract from the UV melting experiments the values of the thermodynamic parameters shown in Table 2. Thus, one has to evaluate the relevance of the extracted apparent thermodynamic parameters in relation to the true ones of the different folded conformers.

\section{The model}

To address the later issue, we consider that two folded states, $\mathbf{1}$ and $\mathbf{1}$ ', coexist with the unfolded state $\mathbf{2}$ during the melting experiments. Instead of only one folding reaction, denoted (1) in the Main Text, two folding processes have now to be taken into account:

$$
\begin{aligned}
& 2=1 \\
& 2=1
\end{aligned}
$$

In reference to the notations used in the Main Text, we designate by $\Delta_{21} H^{0}, \Delta_{21} S^{0}$, and $\Delta_{21} G^{0}$ the standard enthalpy, entropy, and Gibbs free energy associated to the reaction (1), $\Delta_{21^{\prime}} H^{0}, \Delta_{21^{\prime}} S^{0}$, and $\Delta_{21^{\prime}} G^{0}$ the standard enthalpy, entropy, and Gibbs free energy associated to the reaction (2). $\Delta_{21} H^{0}, \Delta_{21} S^{0}, \Delta_{21^{\prime}} H^{0}$, and $\Delta_{21^{\prime}} S^{0}$ are assumed to be constant in the considered temperature range.

We introduce $\alpha$ to measure at chemical equilibrium the proportion of $\mathbf{2}$ in the $\mathbf{1}+\mathbf{1}^{\mathbf{}}+\mathbf{2}$ mixture and $\alpha^{\prime}$ to measure at chemical equilibrium the relative proportion of $\mathbf{1}$ in the mixture of folded conformers $\mathbf{1}+\mathbf{1}$ ':

$$
\alpha(T)=\frac{\alpha^{\prime}(T)}{\alpha^{\prime}(T)+K_{21}(T)}=\frac{\frac{1}{1+\exp \left(-\frac{\Delta_{11^{\prime} G^{0}}}{R T}\right)}}{\frac{1}{1+\exp \left(-\frac{\Delta_{11^{\prime} G^{0}}}{R T}\right)}+\exp \left(-\frac{\Delta_{21} G^{0}}{R T}\right)}
$$

where we introduce $\Delta_{11^{\prime}} H^{0}, \Delta_{11^{\prime}} S^{0}$, and $\Delta_{11^{\prime}} G^{0}$ to respectively designate the standard enthalpy, entropy, and Gibbs free energy associated to the reaction (4):

$$
1=1
$$

In the analysis of a melting experiment relying on a two-level model $(\mathbf{2}=\mathbf{1})$, the experimental melting curve is appropriately normalized to access $\alpha(T)$ that is subsequently 
used to calculate $K_{21}(T)=\frac{1-\alpha(T)}{\alpha(T)}$. Then, the van't Hoff analysis of $K_{21}(T)$ according to $\ln K_{21}(T)=-\frac{\Delta_{21} H^{0}}{R T}+\frac{\Delta_{21} S^{0}}{R}$ yields the values of $\Delta_{21} H^{0}$ and $\Delta_{21} S^{0}$.

When two conformers are present, Eq.(3) provides:

$$
\ln K_{21}^{a p p}(T)=-\frac{\Delta_{21} G^{0}}{R T}+\ln \left[1+\exp \left(-\frac{\Delta_{11^{\prime}} G^{0}}{R T}\right)\right]
$$

where the superscript app was introduced to precise that the two-level model is used to analyze the behavior of the three-component system.

We introduce the respective melting temperatures of $\mathbf{1}$ and $\mathbf{1}^{\prime}: T_{m, 1}=\frac{\Delta_{21} H^{0}}{\Delta_{21} S^{0}}$ and $T_{m, 1^{\prime}}=\frac{\Delta_{21^{\prime}} H^{0}}{\Delta_{21^{\prime}} S^{0}}$. We evaluate the deviation in the standard entropies of reaction between 1 and $1^{\prime}$ by writing $\Delta_{21^{\prime}} S^{0}=x \Delta_{21} S^{0}$ where we take $x \geq 1$. Then, one can easily extract $\Delta_{11^{\prime}} G^{0}$ :

$$
\Delta_{11^{\prime}} G^{0}=(x-1) \Delta_{21} G^{0}+\left(\frac{T_{m, 1^{\prime}}}{T_{m, 1}}-1\right) x \Delta_{21} H^{0}
$$

We can now calculate the apparent thermodynamic parameters $K_{21}^{a p p}(T), \Delta_{21} H^{0, a p p}$ and $\Delta_{21} S^{0, a p p}$, extracted from the application of the two-level model. Three cases have to be envisaged according to the $x$ value.

- $x=1$ :

In relation to the observation of the enthalpy-entropy correlation (vide infra), $\mathbf{1}$ and $\mathbf{1}$ ' here exhibit identical thermodynamic parameters. Then one easily derives:

$$
\begin{aligned}
\ln K_{21}^{\text {app }}(T) & =-\frac{\Delta_{21} G^{0}}{R T}+\ln 2 \\
\Delta_{21} H^{0, a p p} & =\Delta_{21} H^{0} \\
\Delta_{21} S^{0, a p p} & =\Delta_{21} S^{0}+R \ln 2
\end{aligned}
$$

One notices that $\ln K_{21}^{a p p}$ linearly depends on $\frac{1}{T}$. The apparent standard enthalpy $\Delta_{21} H^{0, a p p}$ is equal to the true one, $\Delta_{21} H^{0}$, for $\mathbf{1}$ and $\mathbf{1}$ '. Taking into account the typical orders of magnitude displayed in Table 2 of the Main Text, one notices that $R \ln 2$ is less than $1 \%$ of $\Delta_{21} S^{0}$; as a consequence, one has also $\Delta_{21} S^{0, a p p}=\Delta_{21} S^{0}$ with the present $20 \%$ experimental precision given in Table 2.

- $1<x<1.2$ :

A simple analytical treatment is not possible in that intermediate regime. In contrast, numerical simulations (data not shown) support that the dependence of $\ln K_{21}^{a p p}$ on $\frac{1}{T}$ should be essentially linear in the investigated temperature range. In addition, the extracted apparent thermodynamic values would be equal to the true ones of both foldamers within the $20 \%$ error. 
Assuming that the folded state $\mathbf{1}^{\prime}$ is more stable than the folded state $\mathbf{1}$ at low temperature, one has now typically within $20 \%$ experimental precision:

$$
\begin{aligned}
\ln K_{21}^{\text {app }}(T) & =-\frac{\Delta_{21^{\prime}} G^{0}}{R T} \\
\Delta_{21} H^{0, a p p} & =\Delta_{21^{\prime}} H^{0} \\
\Delta_{21} S^{0, a p p} & =\Delta_{21^{\prime}} S^{0}
\end{aligned}
$$

when $\exp \left(-\frac{\Delta_{11^{\prime}} G^{0}}{R T}\right) \gg 1$ (or $T<T_{i n v}=T_{m, 1^{\prime}}+\frac{T_{m, 1^{\prime}}-T_{m, 1}}{x-1}$; regime of the lowest temperatures) and:

$$
\begin{aligned}
\ln K_{21}^{a p p}(T) & =-\frac{\Delta_{21} G^{0}}{R T} \\
\Delta_{21} H^{0, a p p} & =\Delta_{21} H^{0} \\
\Delta_{21} S^{0, a p p} & =\Delta_{21} S^{0}
\end{aligned}
$$

when $\exp \left(-\frac{\Delta_{11^{\prime}} G^{0}}{R T}\right) \ll 1$ (or $T>T_{i n v}=T_{m, 1^{\prime}}+\frac{T_{m, 1^{\prime}}-T_{m, 1}}{x-1}$; regime of the highest temperatures). One now notices that $\ln K_{21}^{a p p}$ linearly depends on $\frac{1}{T}$ both at low and high temperatures, but with different slopes in the two temperature regimes: The apparent thermodynamic parameters are equal to the ones of the most stable folded conformer in the corresponding temperature regime.

We now take advantage of the linear free energy relation displayed in Figure 9S: In the present series of G4-MB, we experimentally observed:

$$
\Delta_{21} H^{0}\left(J \mathrm{~mol}^{-1}\right)=\rho \Delta_{21} S^{0}\left(J K^{-1} \mathrm{~mol}^{-1}\right)+\eta
$$

(linear regression coefficient: 0.996 ) with $\rho=386 \mathrm{~K}$ and $\eta=3910^{3} \mathrm{~J} \mathrm{~K}^{-1} \mathrm{~mol}^{-1} .{ }^{1}$ Applying the latter free energy relation to $\mathbf{1}$ and $\mathbf{1}$ ', one easily demonstrates: $T_{i n v}=\rho$. Taking into account that we observed $T_{m} \approx 330 \mathrm{~K}$ (see Table 2 of the Main Text), we conclude that we performed the whole extraction of the thermodynamic parameters from the experimental curves in the unique temperature regime $T<\rho$; indeed, the thermodynamic information is essentially contained within the $\left[T_{m}-20 ; T_{m}+20\right]$ temperature range.

Thus, one again notices that $\ln K_{21}^{a p p}$ should linearly depend on $\frac{1}{T}$ in the present series. The apparent thermodynamic parameters should now be equal to the ones of the most stable folded conformer (here $\mathbf{1}^{\prime}$ ).

\footnotetext{
${ }^{1}$ Under such conditions, the difference of the thermodynamic parameters associated to 1 and 1' exceeds the present $20 \%$ experimental precision: $\Delta_{21^{\prime}} S^{0}=x \Delta_{21} S^{0} ; \Delta_{21^{\prime}} H^{0}=x \frac{T_{m, 1^{\prime}}}{T_{m, 1}} \Delta_{21} H^{0}$ with $T_{m, 1^{\prime}}>T_{m, 1}$ because $T_{m, 1^{\prime}}=T_{m, 1}+\frac{(x-1)\left(\rho-T_{m, 1}\right)}{x}$ with $\rho=386 K$ and $T_{m, 1} \simeq 330 K$.
} 


\section{Conclusion}

The following cases are meaningful in relation to the analysis of thermal curves with a two-level model when more than one closed G4-MB is formed:

- If the different folded conformers exhibit identical thermodynamic parameters, the two-level model is analytically appropriate to fit the data extracted from melting experiments and the extracted apparent thermodynamic constants are essentially identical to the true thermodynamic parameters in common to the different foldamers. The system apparently behaves as if it would contain one folded component only with the average spectroscopic features of the different folded states;

- If the difference in the value of the thermodynamic parameters of the different folded states is lower than the experimental error (here 20\%), the dependence of $\ln K_{21}^{a p p}$ on $\frac{1}{T}$ should be essentially linear in the investigated temperature range. In addition, the extracted apparent thermodynamic values would be equal to the true ones of both foldamers within the $20 \%$ error;

- If the difference in the value of the thermodynamic parameters of the different folded states exceeds the experimental error (here $20 \%$ ), one expects to observe a deviation from a linear behavior in the $\ln K_{21}^{a p p}\left(\frac{1}{T}\right)$ curve. In particular, one could be able to extract the true thermodynamic parameters of the different foldamers by linear fit at low and large enough temperatures. Nevertheless, the inversion temperature at which the deviation should appear is here larger than the upper temperature investigated. As a consequence, the two-level model remains here analytically appropriate to fit the data extracted from melting experiments and the apparent thermodynamic parameters should be identical to the true ones of the most stable foldamer. In fact, only one folded state should be significantly populated at any temperature below the inversion temperature and the system again should essentially behave as if it would contain the most stable folded component only. ${ }^{2}$

\footnotetext{
${ }^{2}$ Note that the nature of the observable is here not significant to evidence the presence of several foldamers during melting experiments.
} 


\section{Appendix B: Detailed analysis of the kinetics of associ- ation between 30GMB and its complementary targets}

In this section, we analyze the kinetics of recognition between a molecular beacon belonging to the present G4-MB series and a complementary oligonucleotide. We first examine the kinetic model corresponding to the conversion of the folded G4-MB state to the duplex resulting from association with its target. Then, we report complementary experiments devoted to mechanism analysis. The whole collection of experimental facts is subsequently discussed to discriminate the active mechanism controlling the conversion. The kinetic analysis of the experimental data is described at the end of the section.

\section{The kinetic model}

In this part, we derive the time-dependent evolutions of the concentrations for both mechanisms displayed in Figure 1 of the Main Text.

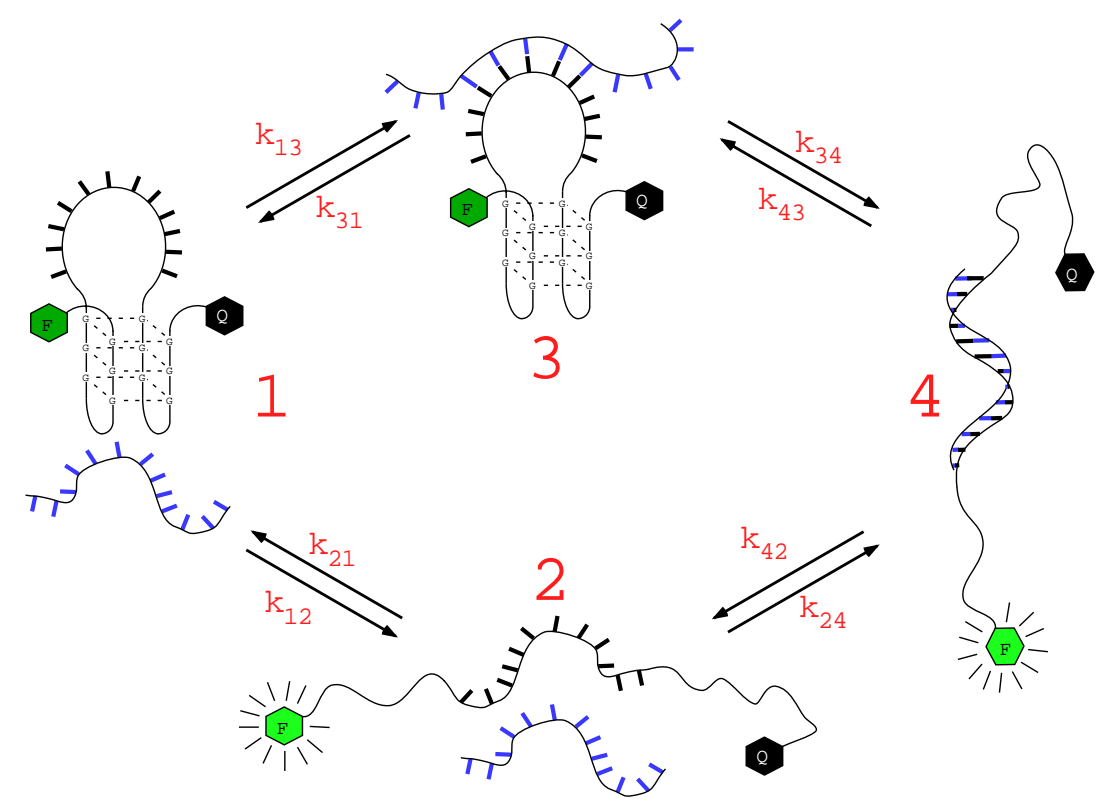

Figure 1. Competing pathways for molecular recognition between an oligonucleotide target and a G4-based molecular beacon (G4-MB). In the upper pathway, partial recognition is followed by invasion that leads to beacon opening. In contrast, binding of the target occurs only after spontaneous opening of the beacon in the lower pathway. The notations: $\mathbf{1}, \mathbf{2}, \mathbf{C}, \mathbf{3}$, and $\mathbf{4}$ stand for the folded G4-MB, the unfolded G4-MB, the complementary single-stranded oligonucleotide, the intermediate resulting from the partial hybridization of $\mathbf{C}$ with the $\mathbf{1}$ loop, and the duplex, respectively. 
In this section, we assume that G4-MB exhibits only one folded state leading to only one set of kinetic and thermodynamic parameters. The behavior alterations resulting from the presence of at least two G4-MB foldamers are discussed in section 3.3.

The opening mechanism 1-2-4 can be written as (17) and the invasion mechanism 1-3-4 as (18).

$$
\begin{array}{rr}
\mathbf{1} \underset{k_{21}}{\stackrel{k_{12}}{\rightleftharpoons}} \mathbf{2} & \mathbf{2}+\mathrm{C} \underset{k_{42}}{\stackrel{k_{24}}{\rightleftharpoons}} \mathbf{4} \\
\mathbf{1}+\mathrm{C} \underset{k_{31}}{\stackrel{k_{13}}{\rightleftharpoons}} \mathbf{3} & \mathbf{3} \underset{k_{43}}{\stackrel{k_{34}}{\rightleftharpoons}} \mathbf{4}
\end{array}
$$

To derive analytical expressions, we assume that the complementary strand $\mathbf{C}$ is in excess compared to the G4-MB derived species. ${ }^{3}$ Then, (17) and (18) can be considered to only involve unimolecular steps, leading to the same simplified mechanism (19):

$$
X \underset{k_{-1}}{\stackrel{k_{1}}{\rightleftharpoons}} Y \underset{k_{-2}}{\stackrel{k_{2}}{\rightleftharpoons}} Z
$$

where $X=\mathbf{1}, Y=\mathbf{2}, Z=\mathbf{4}, k_{1}=k_{12}, k_{-1}=k_{21}, k_{2}=k_{24} C, k_{-2}=k_{42}$ in the case of the opening mechanism 1-2-4 and $X=\mathbf{1}, Y=\mathbf{3}, Z=\mathbf{4}, k_{1}=k_{13} C, k_{-1}=k_{31}, k_{2}=k_{34}$, $k_{-2}=k_{43}$ in the case of the invasion mechanism 1-3-4. In the following, we use the same notation for the species and its concentration.

In a closed system, the set of differential equations governing the time-evolution of the concentrations $X, Y$, and $Z$ related by (19) is given by Eqs.(20-22):

$$
\begin{aligned}
\frac{d X}{d t} & =-k_{1} X+k_{-1} Y \\
\frac{d Y}{d t} & =k_{1} X-\left(k_{-1}+k_{2}\right) Y+k_{-2} Z \\
\frac{d Z}{d t} & =k_{2} Y-k_{-2} Z
\end{aligned}
$$

the solutions of which can be expressed by Eqs.(23-25):

$$
\begin{aligned}
& X(t)=x_{0}+x_{1} e^{\lambda_{1} t}+x_{2} e^{\lambda_{2} t} \\
& Y(t)=y_{0}+y_{1} e^{\lambda_{1} t}+y_{2} e^{\lambda_{2} t} \\
& Z(t)=z_{0}+z_{1} e^{\lambda_{1} t}+z_{2} e^{\lambda_{2} t}
\end{aligned}
$$

where $\lambda_{1}$ and $\lambda_{2}$ designate the nontrivial eigenvalues of the determinant of Eqs.(20-22):

$$
\begin{aligned}
& \lambda_{1}=\frac{1}{2}\left[-\left(k_{1}+k_{-1}+k_{2}+k_{-2}\right)-\sqrt{\left[\left(k_{1}+k_{-1}\right)-\left(k_{2}+k_{-2}\right)\right]^{2}+4 k_{-1} k_{2}}\right] \\
& \lambda_{2}=\frac{1}{2}\left[-\left(k_{1}+k_{-1}+k_{2}+k_{-2}\right)+\sqrt{\left[\left(k_{1}+k_{-1}\right)-\left(k_{2}+k_{-2}\right)\right]^{2}+4 k_{-1} k_{2}}\right]
\end{aligned}
$$

\footnotetext{
${ }^{3}$ As can be seen in the Main Text and below in this section, this hypothesis is always verified in our experiments.
} 
The eigenvalues $\lambda_{1}$ and $\lambda_{2}$ given in Eqs.(26-27) are negative. Thus $-1 / \lambda_{1}$ and $-1 / \lambda_{2}$ can be identified to two relaxation times $\tau_{1}$ (short) and $\tau_{2}$ (long) associated to (19).

Starting from $X$ alone (initial concentration $X_{0}$ ), we eventually compute:

$$
\begin{aligned}
& x_{0}=\frac{k_{-1} k_{-2}}{\lambda_{1} \lambda_{2}} X_{0} ; \quad y_{0}=\frac{k_{1} k_{-2}}{\lambda_{1} \lambda_{2}} X_{0} ; \quad z_{0}=X_{0}-x_{0}-y_{0} \\
& x_{1}=\frac{k_{-1}}{\lambda_{1}-\lambda_{2}}\left[-y_{0}+\left(x_{0}-X_{0}\right) \frac{k_{1}+\lambda_{2}}{k_{-1}}\right] ; \quad y_{1}=x_{1} \frac{k_{1}+\lambda_{1}}{k_{-1}} ; \quad z_{1}=-\left(x_{1}+y_{1}\right) \\
& x_{2}=X_{0}-\left(x_{0}+x_{1}\right) ; \quad y_{2}=-\left(y_{0}+y_{1}\right) ; \quad z_{2}=-\left(z_{0}+z_{1}\right)
\end{aligned}
$$

\section{Complementary experiments}

To discriminate between the mechanisms $\mathbf{1 - 2 - 4}$ and $\mathbf{1 - 3 - 4}$, we envisaged two different approaches. A first series of complementary experiments addressed the mechanism issue by evidencing the formation of the intermediate 3 . In parallel, a second series attempted to perform mechanism discrimination by detailed analysis of the fluorescence relaxation curves associated to G4-MB binding to its targets.

\section{Evidence for the intermediate 3}

To evidence the formation/dissociation of the putative intermediate adduct 3 between $30 \mathrm{GMB}$ and its complementary target, we investigated the reaction of F13ACA with 38G (Table 1 of the Main Text). The first oligonucleotide is a fluorescein-labeled analog of 13ACA, whereas the second one is a G4-MB designed to forbid any disruption of the quadruplex stem: it bears a central loop sequence identical to 30GMB but its longer lateral portions were conceived to form a very stable stem involving five quartets. As expected, the stability of $38 \mathrm{G}$ was considerably larger than that of $30 \mathrm{GMB}\left(T_{m}>90^{\circ} \mathrm{C}\right.$ in the presence of $\mathrm{MgCl}_{2}$; data not shown).

Then we recorded the time evolution of the fluorescence emission from $200 \mathrm{nM}$ F13ACA in $100 \mathrm{mM} \mathrm{KCl} 10 \mathrm{mM} \mathrm{MgCl} 210 \mathrm{mM}$ lithium cacodylate buffer $\mathrm{pH} 7.2$ after sudden addition of 10 equivalents of $38 \mathrm{G}$ at $298 \mathrm{~K}$ (Figures 5Sa and b).

The interaction between F13ACA and 38G was evidenced by a fast $60 \%$ drop in fluorescence emission subsequently followed by a slow minor drift. We attributed the fast decay to the realization of the step 1-3 which did not process further in the 38G case; a monoexponential fit was satisfactory leading to $k_{13}=210^{5} \mathrm{M}^{-1} \mathrm{~s}^{-1}$ as an estimate for the corresponding on-rate for F13ACA:38G pairing. Thereafter, to derive an order of magnitude of the off-rate constant $k_{31}$, we subsequently added a 100-fold excess of 13ACA to the final adduct of the preceding experiment. As shown in Figure 6S, the fluorescence emission from free F13ACA was satisfactorily restituted as expected from 
the displacement of the fluorescent strand by its unlabeled analog. Except for a minor contribution at short times, a monoexponential fit was satisfactory providing $1.610^{-3} \mathrm{~s}^{-1}$ as an estimate for $k_{31}$.

a)

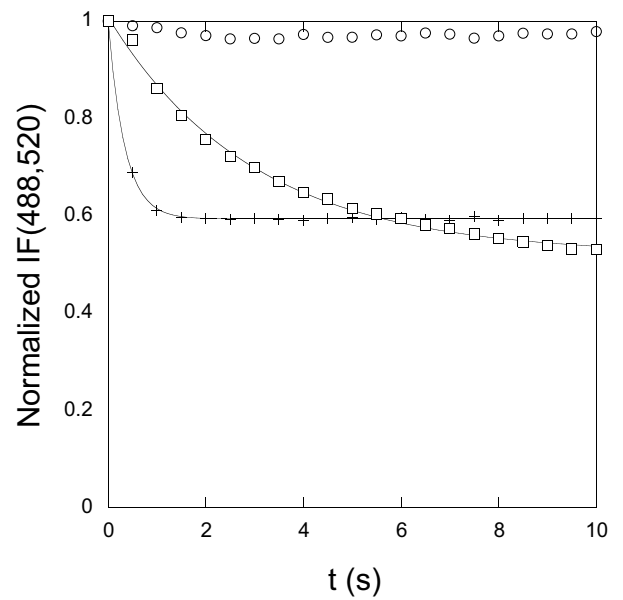

b)

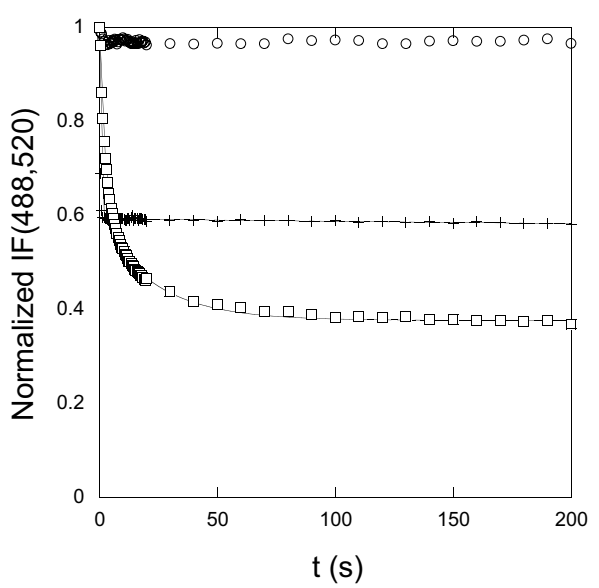

Figure 5S. Short (a) and long (b) time dependence of the fluorescence emission from $200 \mathrm{nM}$ F13ACA after stopped-flow addition of 10 equivalents of 21tel (circles), 38G (squares) or 30con (crosses) at $298 \mathrm{~K}$. Solvent: $100 \mathrm{mM} \mathrm{KCl} 10 \mathrm{mM} \mathrm{MgCl}_{2} 10 \mathrm{mM}$ lithium cacodylate buffer $\mathrm{pH}$ 7.2. $\lambda_{\text {exc }}=488 \mathrm{~nm} ; \lambda_{e m}=520 \mathrm{~nm}$. Markers: experimental points; lines: monoexponential (a) and biexponential (b) fits.

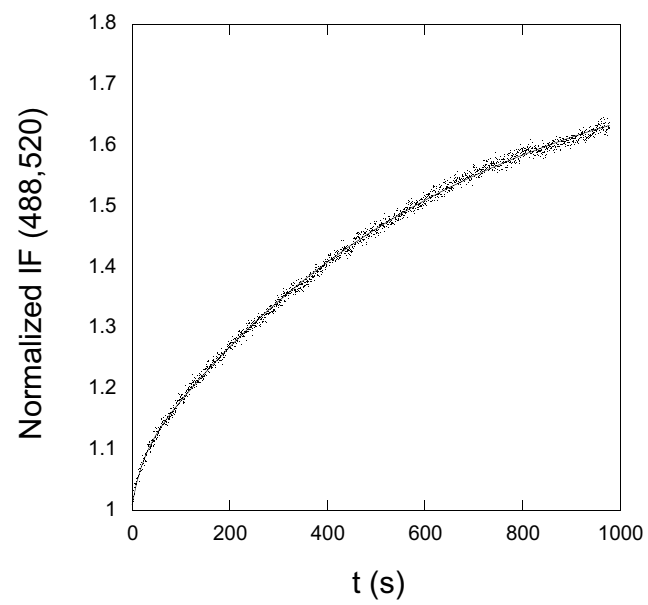

Figure 6S. Time dependence of the fluorescence emission from an equilibrated mixture of 200 $\mathrm{nM}$ F13ACA and $2 \mu \mathrm{M} 38 \mathrm{G}$ after sudden addition of 10 equivalents of 13ACA (i.e. final concentration 13ACA concentration of $20 \mu \mathrm{M}$ ) at $298 \mathrm{~K}$. Solvent: $100 \mathrm{mM} \mathrm{KCl} 10 \mathrm{mM} \mathrm{MgCl}_{2} 10 \mathrm{mM}$ lithium cacodylate buffer $\mathrm{pH}$ 7.2. $\lambda_{e x c}=488 \mathrm{~nm} ; \lambda_{e m}=520 \mathrm{~nm}$. Markers: experimental points; line: monoexponential fit. 
The preceding results suggest that the loop sequence and the complementary strand form a rather stable complex that may exhibit some specific organization. First, we did not observe the formation of such a complex with the much smaller and not complementary 21 tel loop (Figures $5 \mathrm{Sa}$ and $\mathrm{b}$ ). Second, the rather low on-rate constant $k_{13} \simeq 10^{5} \mathrm{M}^{-1} \mathrm{~s}^{-1}$ could reveal a selective nucleation step more than a nonselective hydrophobic collapse expected to be diffusion-controlled $\left(k_{13} \simeq 10^{9} \mathrm{M}^{-1} \mathrm{~S}^{-1}\right)$. Eventually, an estimate of the association constant for 3 formation is given by the ratio $k_{13} / k_{31} \approx 10^{8}$ that does not considerably differ from the association constant for the F13ACA:30con duplex formation, evaluated as $k_{24} / k_{42} \approx 10^{11}$ (vide infra).

\section{Measurements of the rate constants associated to the hybridization of the unfolded beacon}

To complement our derivation of orders of magnitude, we estimated $k_{24}$ and $k_{42}$ by investigating at $298 \mathrm{~K}$ the kinetics of 30con association with 13ACA (step 2-4 in Figure 1). The time dependence of the fluorescence emission from $200 \mathrm{nM} \mathrm{F} 13 \mathrm{ACA}$ in $100 \mathrm{mM} \mathrm{KCl}$ $10 \mathrm{mM} \mathrm{MgCl} 210 \mathrm{mM}$ lithium cacodylate buffer $\mathrm{pH} 7.2$ was recorded after sudden addition of 10 equivalents of 30con (Figures $5 \mathrm{~S}$ a and b). A decrease in fluorescence emission was observed as a function of time. It asymptotically reduces the fluorescein brightness by $40 \%$ and probably reflects a quenching by the 30con guanines facing the fluorescein in the complex. We extracted $1.510^{6} \mathrm{M}^{-1} \mathrm{~s}^{-1}$ as an order of magnitude for the $k_{24}$ rate constant. We also derived $3.210^{-4} \mathrm{~s}^{-1}$ as an estimate of the dissociation rate $k_{42}$ by analyzing the signal evolution arising from adding a 100-fold excess of unlabelled 13ACA to the F13ACA:30con duplex (Figure 7S).

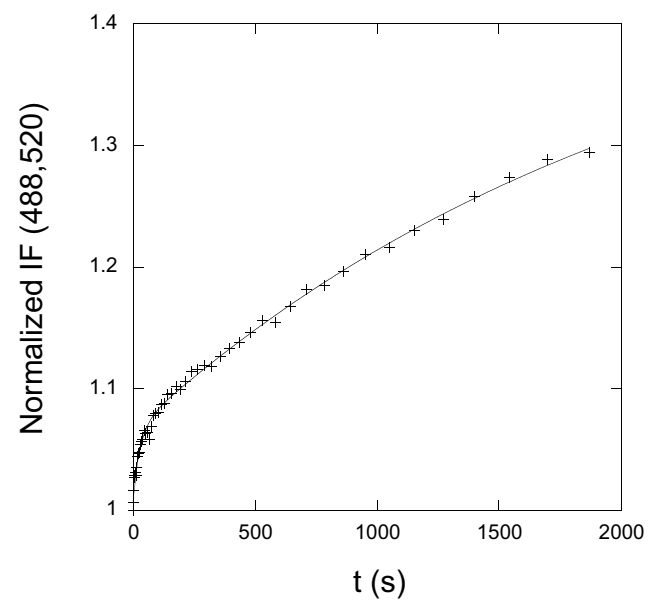

Figure 7S. Time dependence of the fluorescence emission from an equilibrated mixture of 200 nM F13ACA and $2 \mu \mathrm{M} 30 \mathrm{con}$ after sudden addition at $298 \mathrm{~K}$ of 10 equivalent of $30 \mathrm{con}$ in $13 \mathrm{ACA}$ 
(20 $\mu \mathrm{M}$ final concentration in 13ACA) at $298 \mathrm{~K}$. Solvent: $100 \mathrm{mM} \mathrm{KCl} 10 \mathrm{mM} \mathrm{MgCl} 210 \mathrm{mM}$ lithium cacodylate buffer $\mathrm{pH}$ 7.2. $\lambda_{e x c}=488 \mathrm{~nm} ; \lambda_{e m}=520 \mathrm{~nm}$. Crosses: experimental points; line: biexponential fit.

\section{Kinetic characterization of the overall process: sequence and concentration dependence}

In principle, the dependence of the characteristic relaxation times associated to G4-MB opening could bring valuable insights to facilitate mechanism discrimination (vide infra). Figures $8 \mathrm{Sa}, \mathrm{b}$, and c display the time evolution of the fluorescence emission from 200 nM 30GMB after addition of different amounts of 13ACA, 13ACC, or 13AAA in $100 \mathrm{mM}$ $\mathrm{KCl} 10 \mathrm{mM} \mathrm{MgCl} 210 \mathrm{mM}$ lithium cacodylate buffer $\mathrm{pH} 7.2$ at $298 \mathrm{~K}$.

a)

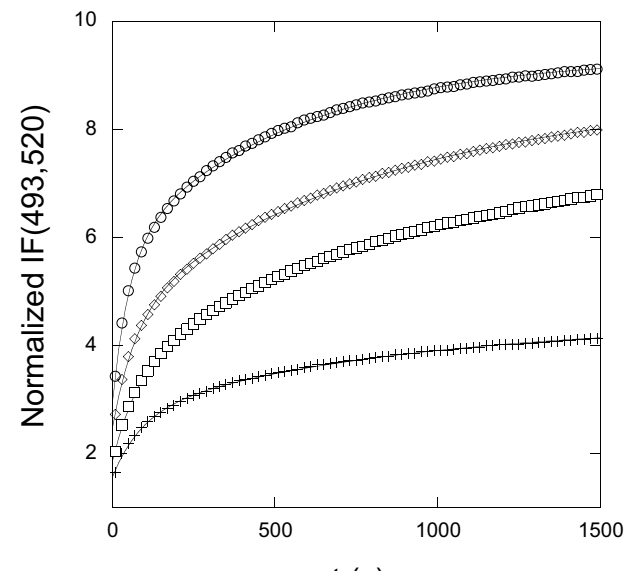

$t(s)$ b)

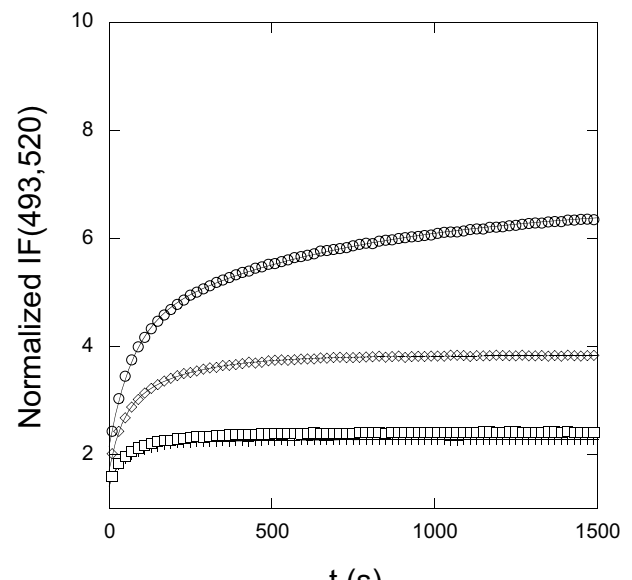

$\mathrm{t}(\mathrm{s})$

c)

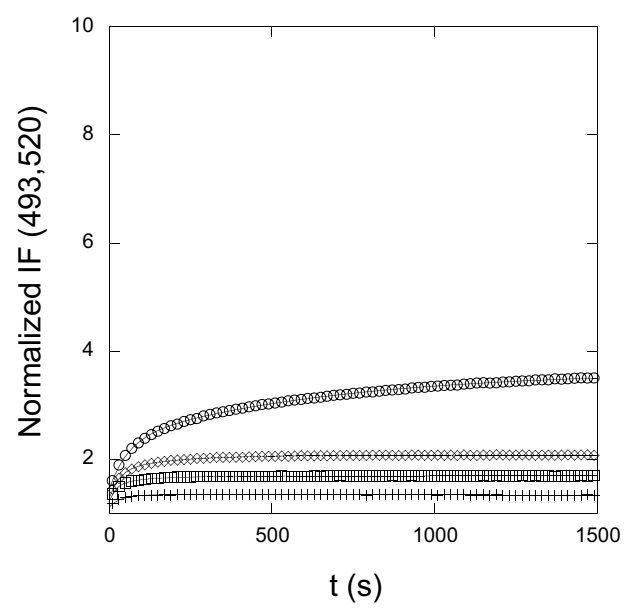

Figure 8S. Change in the fluorescence emission from $200 \mathrm{nM} 30 \mathrm{GMB}$ with respect to time after sudden addition at $298 \mathrm{~K}$ of 0.5 (crosses), 1 (squares), 5 (diamonds), or 50 (circles) equivalents of 13ACA (a), 13ACC (b), and 13AAA (c). $\lambda_{e x c}=493 \mathrm{~nm} ; \lambda_{e m}=520 \mathrm{~nm}$. Solvent: $100 \mathrm{mM} \mathrm{KCl}$ $10 \mathrm{mM} \mathrm{MgCl} 210 \mathrm{mM}$ lithium cacodylate buffer $\mathrm{pH}$ 7.2. Markers: experimental points; lines: 
biexponential fits.

Figure 8Sa displays the results obtained in the series of experiments with 13ACA. As also shown in Table 4S, two time scales are associated to the interaction of 30GMB with this complementary strand. In a first kinetic regime, the signal increases in the 50-100 s range, whereas in a second one, the fluorescence evolution is about ten times slower (on a 500-1000 s time scale). As anticipated, the final fluorescence emission increases with an increase of the concentration $C$ in the complementary target. Table $4 \mathrm{~S}$ shows that the amplitudes $a_{a}$ and $a_{b}$ associated to both kinetic regimes are essentially independent on $C$. In contrast, the relaxation times associated to both kinetic regimes decrease by a factor 2 when using from 1 to 50 equivalents in 13ACA.

The Figures $8 \mathrm{Sb}$ and $\mathrm{c}$ show the corresponding behavior when either 13ACC or 13AAA was added to 30GMB. At the largest concentrations in complementary strand, one finds again the behavior that was observed with 13ACA, however with some delay in the concentration of complementary target required to observe a given time evolution. In contrast, we only observe a rather fast slight increase of fluorescence emission at the lowest investigated concentrations in complementary strand.

Table 4S. Relaxation times and amplitudes obtained from biexponential analysis (Eq.(15) in the Main Text) of the time evolution of the fluorescence emission from $200 \mathrm{nM}$ 30GMB after sudden addition at $298 \mathrm{~K}$ of $0.5,1,5$, or 50 equivalents of $13 \mathrm{ACA}$. $\lambda_{e x c}=493 \mathrm{~nm} ; \lambda_{e m}=520 \mathrm{~nm}$. Solvent: $100 \mathrm{mM} \mathrm{KCl} 10 \mathrm{mM} \mathrm{MgCl} 210 \mathrm{mM}$ lithium cacodylate buffer $\mathrm{pH}$ 7.2.

\begin{tabular}{lcccc} 
Number of equivalents & $\tau_{a}(\mathrm{~s})$ & $a_{a}(\%)$ & $\tau_{b}(\mathrm{~s})$ & $a_{b}(\%)$ \\
\hline & & & & \\
0.5 & 91 & 40 & 862 & 60 \\
1 & 87 & 30 & 971 & 70 \\
5 & 73 & 30 & 826 & 70 \\
50 & 53 & 40 & 543 & 60 \\
& & & & \\
\hline
\end{tabular}

\section{Discussion}

We successively considered two types of arguments to discriminate between the mechanisms 1-2-4 and 1-3-4. 


\section{Evidence for the intermediate 3}

The above complementary experiments showed that a 13ACA:G4-MB intermediate 3 forms and can be observed when G4-MB opening is impeded. It was also possible to derive the orders of magnitude of i) the on-rate constant $k_{13}$ and ii) the thermodynamic constant $K_{13}$ corresponding to 3 formation. Over micromolar concentrations in complementary strand $C$, such values are compatible with the fast realization of the equilibrium 1-3 with respect to the steps 1-2 and 3-4 $\left(k_{13} C>k_{12}\right.$ and $k_{13} C>k_{34} ;$ vide infra $)$. This result would apply for all the oligonucleotide sequences that were investigated since $k_{13}$ should not considerably depend on the presence of any mismatch. In addition, the extent of reaction 1-3 at equilibrium above micromolar concentrations $C$ should favor the intermediate 3 during G4-MB opening. This result is expected for the matched sequence where $K_{13} C \gg 1$ under such conditions. It probably also applies for mismatched sequences. Indeed $K_{13}$ should not be lower than $10^{8}$ by more than two orders of magnitude in these cases.

\section{Temporal evolution of the concentrations}

We successively considered the two possible mechanisms, 1-2-4 and 1-3-4, with particular emphasis on the dependence of the relaxation times $\tau_{1}$ and $\tau_{2}$ on the concentration in complementary strand $C$. We then examined whether the analysis of the temporal evolution of the fluorescence emission from the G4-MB could be used to discriminate the mechanisms 1-2-4 and 1-3-4.

First possibility: the mechanism 1-2-4 is operative The relaxation times of the individual steps 1-2 and 2-4 can be evaluated by using the values of $k_{12}, k_{21}, k_{24}$ and $k_{42}$ given in the Main Text and derived above. The relaxation time of the step 1-2, $\tau_{12}$, is in the $100 \mathrm{~s}$ range whereas the relaxation time of the step $\mathbf{2 - 4}, \tau_{24}$, is in the $1 \mathrm{~s}$ range at the relevant micromolar concentrations in complementary strands (see Eqs. $(8,12)$ in the Main Text). If the mechanism 1-2-4 is operative, one should thus conclude that the step 1-2 is rate-limiting under the present experimental conditions. Then, $\left(k_{1}+k_{-1}\right) \ll\left(k_{2}+k_{-2}\right)$ and at first order from Eqs. $(26,27)$ :

$$
\begin{aligned}
& \frac{1}{\tau_{1}}=-\lambda_{1} \approx k_{2}+k_{-2}=k_{24} C+k_{42} \\
& \frac{1}{\tau_{2}}=-\lambda_{2} \approx k_{1}+k_{-1}\left(1-\frac{1}{1+\frac{k_{-2}}{k_{2}}}\right)=k_{12}+k_{21}\left(\frac{1}{1+\frac{k_{24} C}{k_{42}}}\right)
\end{aligned}
$$

Taking into account the orders of magnitude for the values of $k_{12} \approx 10^{-2}-10^{-3} \mathrm{~s}^{-1}, k_{21} \approx$ $10^{-1}-10^{-2} \mathrm{~s}^{-1}, k_{24} \approx 10^{6} \mathrm{M}^{-1} \mathrm{~s}^{-1}$, and $k_{42} \approx 10^{-5} \mathrm{~s}^{-1}$ as well as the overmicromolar 
concentration $C$ in complementary strand at the largest investigated concentrations, one should observe under the present experimental conditions the temporal behavior expressed by Eqs.(33-34):

$$
\begin{aligned}
& \frac{1}{\tau_{1}} \approx k_{24} C \\
& \frac{1}{\tau_{2}} \approx k_{12}
\end{aligned}
$$

Above micromolar concentrations in complementary strand, the short relaxation time $\tau_{1}$ should be lower than $1 \mathrm{~s}$ and would not be observed during the present series of experiments. In contrast, the long relaxation time $\tau_{2}$ should i) be in the $70-500 \mathrm{~s}$ range $\left(k_{12 a}=1.910^{-3} \mathrm{~s}^{-1}\right.$ and $\left.k_{12 b}=1.410^{-2} \mathrm{~s}^{-1}\right)$; ii) not depend on the concentration in complementary strand $C$.

Second possibility: the mechanism 1-3-4 is operative The relaxation time $\tau_{13}$ of the step 1-3 can be evaluated from the values of $k_{13}$ and $k_{31}$. In contrast, $k_{43}$ and $k_{34}$ could not be independently measured as the other rate constants extracted in the present investigation. Hence it is not possible to evaluate a priori the order of magnitude of the relaxation time $\tau_{34}$ of the step 3-4. At that point, we assume that the rate limiting step under the present experimental conditions should be 3-4 (this assumption will have to be validated later). Then, $\left(k_{1}+k_{-1}\right) \gg\left(k_{2}+k_{-2}\right)$ and from Eqs. $(26,27)$ at first order:

$$
\begin{aligned}
& \frac{1}{\tau_{1}}=-\lambda_{1} \approx\left(k_{1}+k_{-1}\right)=\left(k_{13} C+k_{31}\right) \\
& \frac{1}{\tau_{2}}=-\lambda_{2} \approx k_{-2}+k_{2}\left(1-\frac{1}{1+\frac{k_{1}}{k_{-1}}}\right)=k_{43}+k_{34}\left(\frac{\frac{k_{13} C}{k_{31}}}{1+\frac{k_{13} C}{k_{31}}}\right)
\end{aligned}
$$

Above micromolar concentrations in complementary strand, the short relaxation time $\tau_{1}$ should be lower than $10 \mathrm{~s}$. Then the 100-1000 s relaxation times in Figures 8Sa, b, and c could only correspond to the long relaxation time $\tau_{2}$ associated to the step $\mathbf{3 - 4}$.

In contrast to the preceding situation, $k_{13} C$ could not systematically exceed $k_{31}$ in the investigated $C$ range, due to the lower stability of the intermediate 3 with regard to the duplex 4. At the highest investigated $C$ concentrations, $k_{13} C \gg k_{31}$ and the longest relaxation time $\tau_{2}^{-1} \approx k_{43}+k_{34}$ from Eq.(36). In contrast, $\tau_{2}^{-1} \approx k_{43}$ for the lowest $C$ values.

In fact, a crude derivation of $k_{43}$ and $k_{34}$ results from the observation that 30GMB opening is not complete even in the presence of the matched sequence 13ACA. Indeed Figure 5b (see Main Text) shows that the pairing of unfolded 30GMB with its complementary strand is accompanied by an increase of fluorescence emission by about $20 \%$ 
whereas the fluorescence emission of the mixture of folded 30GMB with 13ACA below $30^{\circ} \mathrm{C}$ is identical to the one of unfolded 30GMB (Figure 6). The simplest explanation to the latter observation consists in assuming that the system still contains some intermediate 3 below $30^{\circ} \mathrm{C}$ (by using the relative fluorescence intensities shown in Figures 5b and 6 at $30^{\circ} \mathrm{C}$ and $80^{\circ} \mathrm{C}$, we evaluate the relative proportions to about $80 \%$ of duplex 4 , and $20 \%$ of intermediate $\mathbf{3}$ ). Then the thermodynamic constant associated to the duplex 4 formation, $K_{34}=\frac{k_{34}}{k_{43}}$, is not much more than one under the present experimental conditions. In the case of 30GMB:13ACA, we retained $K_{34}=\frac{k_{34}}{k_{43}} \approx 1$ as an order of magnitude. As $k_{43} \lesssim \tau_{2}^{-1} \lesssim k_{43}+k_{34}$, we derived from Table $4 \mathrm{~S}: k_{34} \approx 10^{-3} \mathrm{~s}^{-1}$ and $k_{43} \approx 10^{-3} \mathrm{~s}^{-1}$ as crude estimates. Such values are in line with the corresponding rate constants that were observed for the step 1-2. Indeed both steps are associated to G4-MB opening.

Finally, if the mechanism $\mathbf{1 - 3 - 4}$ is operative, i) the rate-limiting step is effectively $\mathbf{3 - 4}$ during the conversion from the state 1 to the state 4 ; ii) the short relaxation time $\tau_{1}$ should be lower than $10 \mathrm{~s}$ above micromolar concentrations in complementary strand and could be hardly observed at the largest $C$ values during the present series of experiments; iii) the long relaxation time $\tau_{2}$ should be in the $10^{3} \mathrm{~s}$ range and could slightly depend on the concentration in complementary strand $C$ in the rather narrow range of values $k_{43} \lesssim \tau_{2}^{-1} \lesssim 2 k_{43}$.

Analysis of the experimental results We successively examine the amplitude and the time-dependence of the fluorescence evolution from 30GMB.

Analysis of the amplitude of the fluorescence emission from 30GMB as a function of time In principle, the analyses of the amplitude of the fluorescence emission as a function of time may provide some insight into the nature of the mechanism, $\mathbf{1 - 2 - 4}$ or $\mathbf{1 - 3 - 4}$.

If the mechanism 1-2-4 is relevant, one would directly observe the conversion of the closed beacon 30GMB 1 to the duplex 4 at the $\tau_{2}$ time scale. In fact, the signal increase by a factor 8 that is observed in Figure $8 \mathrm{Sa}$ at 50 equivalents in 13ACA would be reasonably in line with this interpretation. First, 30GMB denaturation was shown to be associated by a 16-fold fluorescence increase resulting from the levelling-off of the quenching of fluorescein emission by dabcyl (see Main Text). Duplex formation from unfolded 30G and F13ACA was secondly shown to be accompanied by a $40 \%$ fluorescence drop (vide supra). If the latter drop reflects some fluorescein quenching associated to 13ACA-30G duplex formation, the 1-4 30GMB conversion should be associated to a fluorescence increase of fluorescein in the $16 \times 0.4$ range.

If the mechanism 1-3-4 is active, one would reach a fast 1-3 equilibrium followed by a slow conversion of the intermediate 3 to the duplex 4 at the $\tau_{2}$ time scale. Then 
one should deduce from the 8-fold signal increase that is observed in Figure 8Sa that the intermediate 3 and the closed beacon 30GMB 1 would exhibit a similar brightness. This would not contradict the expectations if one considers that the interaction of the complementary target with the 30GMB loop does not considerably affect the relative positions of fluorescein and dabcyl in the G4-MB moiety. Given the relative positions of the labels with regards to the loop, the latter assumption appears reasonable.

Thus it does not appear possible to distinguish between the mechanisms 1-2-4 and 1-3-4 on the basis of the amplitude analyses.

Analysis of the temporal evolution of the fluorescence emission from $G 4-M B$ The values of the relaxation times $\tau_{2}$ observed in Figures $8 \mathrm{Sa}, \mathrm{b}$, and c cannot be used to discriminate among the mechanisms 1-2-4 and 1-3-4. Indeed similar values could be expected in both cases by appropriately attributing the values of the rate constants.

However, as explained above, the study of the dependence of $\tau_{2}$ on $C$ could be used to make the discrimination. In the $13 \mathrm{ACA}$ case, Table $4 \mathrm{~S}$ shows that the two relaxation times $\tau_{2 a}$ and $\tau_{2 b}$ effectively decrease by a factor 2 when the concentration $C$ in complementary strand is increased by a factor 10 (entries 5 and 50 equivalents in $13 \mathrm{ACA}^{4}$ ). This observation would be both qualitatively and quantitatively in line with the mechanism 1-3-4 to control unfolding of (at least) two conformers of 30GMB in comparable amounts (vide supra). In fact such an explanation would additionally agree with i) the kinetic measurements of 30GMB unfolding. Indeed, the factor 10 found between $\tau_{2 a}$ and $\tau_{2 b}$ satisfactorily compare with the corresponding factor between the two relaxation times that were experimentally found to be associated to 30 GMB unfolding $\left(\tau_{12 a}=530 \mathrm{~s}\right.$ and $\tau_{12 b}=70 \mathrm{~s}$ ); ii) the kinetic behavior in the case of mismatched sequences $13 \mathrm{ACC}$ and 13AAA. In the latter case, one could imagine that the less stable and possibly most reactive $30 \mathrm{GMB}$ conformer(s) preferentially react(s): the relaxation time should be small at the lowest concentrations $C$ in complementary strand. The more stable and possibly less reactive $30 \mathrm{GMB}$ conformer(s) would then react at larger concentrations $C$ leading to behavior comparable to the ones observed in the 13ACA case at lower concentrations $C$.

\section{Conclusion}

All together, the observation of an intermediate 3 in the case of a G4-MB that cannot open, associated to the dependence of the relaxation times for 30GMB opening with the concentration in complementary strand incline to favor the mechanism 1-3-4 as opera-

\footnotetext{
${ }^{4}$ The experiments in which 0.5 or 1 equivalent of $13 \mathrm{ACA}$ is added to $30 \mathrm{GMB}$ cannot be easily analyzed. Indeed, the absence of excess in the complementary strand makes difficult the analysis of the characteristic times of the biexponential fit (see Eq.(9) in the Main Text). In addition, the rather long relaxation time $\tau_{13}$ interferes with the fastest relaxation time of the step 3-4 under such conditions.
} 
tive in the case of the matched complementary oligonucleotide 13ACA. ${ }^{5}$ Consequently, the present experimental results suggest the following mechanism to be active during hybridization between a folded G4-MB such as 30GMB, and an oligonucleotide target complementary to its loop: i) the target first wraps onto a part of the probe domain of G4-MB which remains closed; ii) an increase in the number of zipped base pairs subsequently leads to quadruplex opening, in a manner analogous to the mechanism described for regular MB.[3] Noticeably, the time scale that is associated to the second step 3-4 does not significantly depart from the time scale that is associated to the spontaneous opening of 30GMB. Hence the zipping process arising from the duplex formation does not act as a strong driving force for quadruplex disruption.

\section{Kinetic Analysis of the Experimental Data}

The kinetic situations encountered during the present study are displayed in Figure 1. They correspond to kinetic laws that can be grouped in two categories: isomerizations and bimolecular reactions. The first category was appropriate to extract the rate constants $k_{12}$ and $k_{21}$, whereas the second was used to extract orders of magnitude of $k_{24}, k_{42}, k_{13}$, and $k_{31}$.

The isomerizations were modeled by considering a process in which a reactant $\mathbf{R}$ (initial concentration $R_{0}$ ) yields a product $\mathbf{P}$ (initial concentration 0 ):

$$
\mathbf{R} \underset{k_{-}}{\stackrel{k_{+}}{\rightleftharpoons}} \mathbf{P}
$$

Then, the time evolution of the concentrations can be written:

$$
R(t)=R_{\infty}+\left(R_{0}-R_{\infty}\right) \exp (-t / \tau)=R_{0}-P(t)
$$

with

$$
\tau=\frac{1}{k_{+}+k_{-}}
$$

and where $R_{\infty}$ designates the concentration in $\mathbf{R}$ at equilibrium. During the present series of experiments, one of the rate constants, $k_{+}$or $k_{-}$, was set at a much larger value than the other so as to directly extract it from the relaxation time $\tau$.

For bimolecular reactions, we considered that we started from a mixture of reactants $\mathbf{R}$ and $\mathbf{R}^{*}$ (respective initial concentrations $R_{0}$ and $R_{0}^{*}$ ) yielding a product $\mathbf{P}$ (initial concentration 0$)$ :

$$
\mathbf{R}+\mathbf{R}^{*} \stackrel{k_{+}}{\underset{k_{-}}{\rightleftharpoons}} \quad \mathbf{P}
$$

\footnotetext{
${ }^{5}$ In contrast, the present data cannot exclude the mechanism 1-2-4 to play some role in the pairing of the mismatched sequences $13 \mathrm{ACC}$ and 13AAA with 30GMB.
} 
Then, the time evolution of the concentrations in $\mathbf{R}, \mathbf{R}^{*}$, and $\mathbf{P}$ can be written:

$$
\begin{aligned}
R(t) & =R_{\infty}+\left(R_{0}-R_{\infty}\right) \frac{\exp (-t / \tau)}{1+k_{+} \tau P_{\infty}[1-\exp (-t / \tau)]}=R_{0}-P(t) \\
R^{*}(t) & =R_{0}^{*}-P(t)
\end{aligned}
$$

with

$$
\tau=\frac{1}{k_{+}\left(R_{0}+R_{0}^{*}-2 P_{\infty}\right)+k_{-}}
$$

In the preceding equations, $R_{\infty}, R_{\infty}^{*}$ and $P_{\infty}$ stand for the concentrations at equilibrium in $\mathbf{R}, \mathbf{R}^{*}$, and $\mathbf{P}$, respectively. If one of the species, $\mathbf{R}$ or $\mathbf{R}^{*}$, is in excess (here $\mathbf{R}^{*}$ for illustration), Eq.(41) adopts the dependence shown in Eq.(38) with:

$$
\tau=\frac{1}{k_{+} R_{0}^{*}+k_{-}}
$$

In the course of this study, we systematically adopted appropriate experimental conditions to remain in the preceding regime of approximation. As for isomerizations, one of the terms contained in $\tau, k_{+} R_{0}^{*}$ or $k_{-}$, was much larger than the other during the present series of experiments so as to directly extract its value from the relaxation time $\tau$.

Eventually, we also performed displacement experiments in which the unlabeled oligonucleotide 13ACA $(\mathbf{A})$ replaced the F13ACA labeled analog $\left(\mathbf{A}^{*}\right)$ in the intermediate $\mathbf{3}$ or in the duplex $\mathbf{4}\left(\mathbf{A B}\right.$ or $\left.\mathbf{A}^{*} \mathbf{B}\right)$. The corresponding kinetic scheme can be written:

$$
\begin{array}{ccc}
\mathbf{A}^{*} \mathbf{B} & \underset{k_{-1}}{\rightleftharpoons} & \mathbf{A}^{*}+\mathbf{B} \\
\mathbf{A}+\mathbf{B} & \underset{k_{-2}}{\rightleftharpoons} & \mathbf{A B}
\end{array}
$$

If the solution initially only contains $\mathbf{A}^{*} \mathbf{B}$ (concentration $A_{t o t}^{*}$ ), the addition of $\mathbf{A}$ in excess leads to the complete conversion of $\mathbf{A}^{*} \mathbf{B}$ into $\mathbf{A B}$ at equilibrium. When the concentrations are chosen to make the dissociation of $\mathbf{A}^{*} \mathbf{B}$ rate-limiting with regards to the subsequent formation of $\mathbf{A B}$, the time dependence of the $\mathbf{A}^{*}$ concentration is given by:

$$
A^{*}(t)=A_{t o t}^{*}\left[1-\exp \left(-k_{1} t\right)\right]
$$

For each of our experiments, the recorded signal in fluorescence emission, $S(t)$, is the sum of all the individual contributions from the present species i. In the present range of absorbance values, each contribution $s_{i}(t)$ is proportional to the brightness $Q_{i}$ and the concentration $C_{i}(t)$ of the considered species i. Thus, we always used Eq.(48), with the 
corresponding expression of the relaxation time, to analyze the temporal evolution of the fluorescence emission when monoexponential fitting was appropriate:

$$
S(t)=\sum_{i} s_{i}(t)=S_{\infty}+\left(S_{0}-S_{\infty}\right) \exp (-t / \tau)
$$

where $S_{0}$ and $S_{\infty}$ respectively designate the initial and final fluorescence signal. In contrast, we adopted the biexponential fitting law:

$$
S(t)=\sum_{i} s_{i}(t)=S_{\infty}+a_{a} \exp \left(-t / \tau_{a}\right)+a_{b} \exp \left(-t / \tau_{b}\right)
$$

when the monoexponential law failed to fit the experimental data. 


\section{Appendix C: Electrophoretic measurements}

We performed capillary electrophoresis experiments on the $30 \mathrm{G}$ sequence. They were directed towards the investigation of:

- the possible presence of several $30 \mathrm{G}$ folded states in the potassium containing buffers;

- the significance of the $\mathrm{Mg}^{2+}$ cation on the $30 \mathrm{G}$ electrophoretic mobility;

- the comparison of the rate constants associated to the folding/unfolding processes within the mixture of $30 \mathrm{G}$ folded states.

Figure 9-11S display the electropherograms by capillary electrophoresis of 30G and 30con samples, equilibrated in different buffers, and separated in columns conditioned with different buffers.

Figures 9a and 9b compare the electropherograms of 30G in potassium and lithium containing buffers, either in the absence or in the presence of magnesium cation. The electropherogram of 30con, which do not form any motif, was used as a 30G reference.

a)

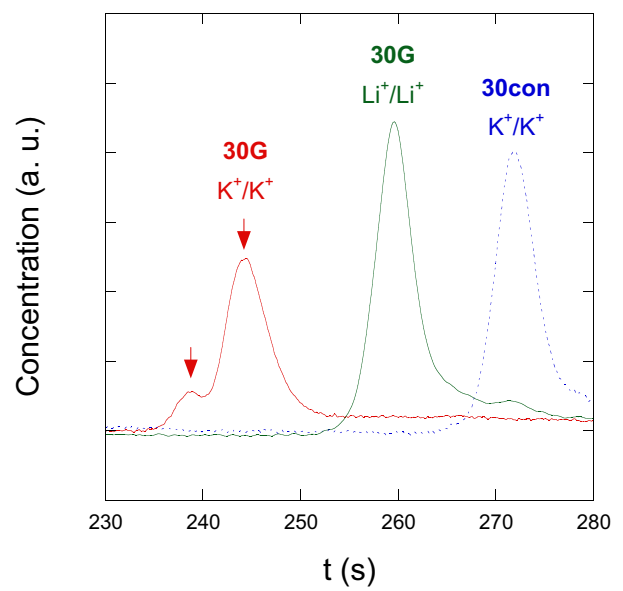

b)

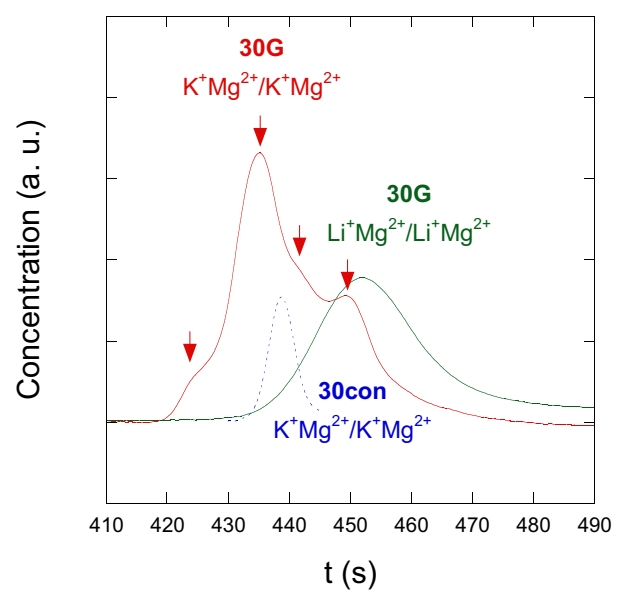

Figure 9S. Electropherograms of $30 \mathrm{G}$ in potassium and lithium containing buffers using the electropherogram of the G4-nonforming 30con sequence as a reference, either in the absence (a) or in the presence (b) of magnesium cation. The notation cation/cation below the name of the sequence indicates the nature of the buffer cation in the injected oligonucleotide sample and in the column, respectively. Buffers: $220 \mathrm{mM} \mathrm{K}$ (or Li) Hepes pH 7.45 or $220 \mathrm{mM} \mathrm{K}$ (or Li) Hepes pH 7.45 containing $10 \mathrm{mM} \mathrm{Mg}{ }^{2+}$; $\mathrm{T}=298 \mathrm{~K}$; Applied voltage: $10 \mathrm{kV}$; concentration of the injected samples $0.2 \mathrm{mM}$.

One notices: 
- the presence of a unique peak for $30 \mathrm{G}$ in lithium containing buffers or for 30con in potassium containing buffers. This observation is in line with the unique unfolded state anticipated from the melting experiments for the corresponding sequences under the present experimental conditions;

- the presence of several peaks for $30 \mathrm{G}$ in potassium containing buffers that suggests the existence of several $30 \mathrm{G}$ foldamers under the present experimental conditions. In the absence of magnesium cation, one observes two peaks; in contrast, four peaks (two large and two smaller) can be recognized in the presence of magnesium. One may additionally note the absence of any residual peak corresponding to the unfolded state that should be eluted like 30con; this observation is in line with the existence of $30 \mathrm{G}$ folded states only at room temperature, expected from melting experiments;

- the alterations of electrophoretic mobility. In agreement with their smaller size, the folded states migrate faster than the unfolded ones. In addition, the migration is considerably smaller in the presence of magnesium cation which denotes a stronger interaction of the negatively charged oligonucleotidic sequences with $\mathrm{Mg}^{2+}$ than with the monovalent cations.

Figure 10S is relevant to analyze the folding process that occurs when the unfolded $30 \mathrm{G}$ state initially contained in a lithium containing buffer is injected in a column conditioned with a potassium containing buffer. At the time scale of the electropherograms (450 s), one notices that the injected sample reached the equilibrium state. This observation:

- is in line with the results displayed in Figure 4a of the Main Text. During the latter experiment, the folding time scale was expected to lie in the $4-25$ s range which indeed is smaller than the elution time $(450 \mathrm{~s}) ;^{6}$

- suggests that the distribution of the folding rate constants among the foldamer population is rather narrow. Indeed the slowest folding time scale among the foldamer population should be lower than 200 s. All together, the different experiments suggest that the $4-200$ s range at most for the folding time scale of $30 \mathrm{G}$.

\footnotetext{
${ }^{6}$ It was impossible to decrease the elution time with the present experimental set up.
} 


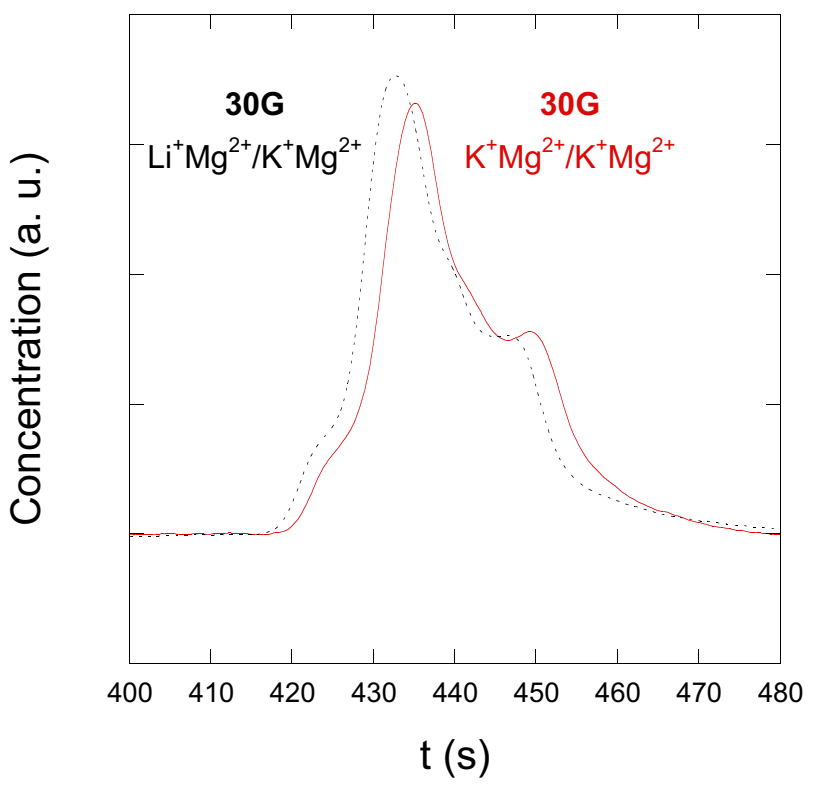

Figure 10S. Electropherograms of 30G in potassium containing buffers in the presence of magnesium cation. The notation cation/cation below the name of the sequence indicates the nature of the buffer cation in the injected oligonucleotide sample and in the column respectively. Buffer: 220 mM K (or Li) Hepes pH 7.45 containing $10 \mathrm{mM} \mathrm{Mg}^{2+}$; $\mathrm{T}=298 \mathrm{~K}$; Applied voltage: $10 \mathrm{kV}$; concentration of the injected samples $0.2 \mathrm{mM}$.

Figure $11 \mathrm{~S}$ analyzes the unfolding process that takes place when the equilibrated mixture of $30 \mathrm{G}$ foldamers in a potassium containing buffer is injected in a column conditioned with a lithium containing buffer. At the time scale of the electropherogram (450 s), one again notices that the injected sample reached the unfolded equilibrium state. This observation:

- is in reasonable agreement with the results displayed in Figure 4b of the Main Text. During the latter experiment, the unfolding time scale was observed in the $70-500$ s range and the latter values are essentially smaller than the elution time $(450 \mathrm{~s}) ;^{7}$

- suggests that the distribution of the unfolding rate constants among the foldamer population is rather narrow. In particular, one notices the absence of any shoulder in the observed peak. Thus the slowest unfolding time scale within the foldamer population should not exceed about $450 \mathrm{~s}$. All together, the different experiments suggest the $70-500 \mathrm{~s}$ range at most for the unfolding time scale of $30 \mathrm{G}$.

\footnotetext{
${ }^{7}$ It was impossible to decrease the elution time with the present experimental set up.
} 


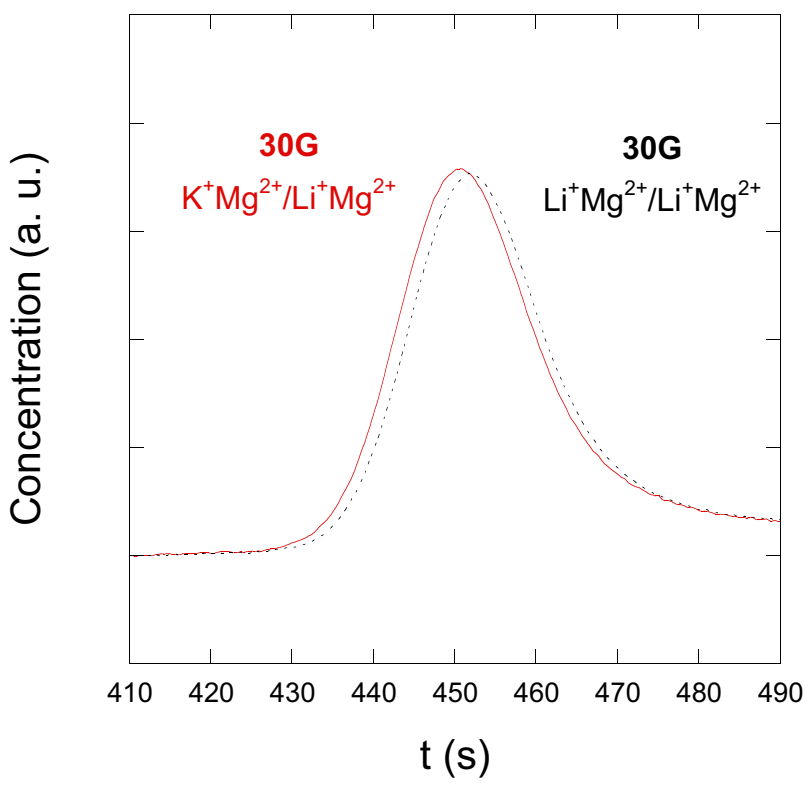

Figure 11S. Electropherograms of 30G in lithium containing buffers in the presence of magnesium cation. The notation cation/cation below the name of the sequence indicates the nature of the buffer cation in the injected oligonucleotide sample and in the column respectively. Buffer: $220 \mathrm{mM} \mathrm{K}$ (or Li) Hepes pH 7.45 containing $10 \mathrm{mM} \mathrm{Mg}^{2+}$; $\mathrm{T}=298 \mathrm{~K}$; applied voltage $=12.5$ $\mathrm{kV}$; concentration of the injected samples $0.2 \mathrm{mM}$.

\section{Conclusion}

We evidenced the presence of several $30 \mathrm{G}$ folded states at room temperature. At the same time, the combination of the results from the kinetic experiments relying on fluorescence emission and capillary electrophoresis led us to conclude that the rate constants of the different $30 \mathrm{G}$ foldamers are most probably contained in a range covering about one order of magnitude. This conclusion is in line with the results displayed in Figure 4 of the Main Text and the corresponding analyses.

The preceding electrophoretic measurements bear also some significance to evaluate the distribution of the thermodynamic constant associated to the folding process within the population of $30 \mathrm{G}$ folded states: The observation that the rate constants associated to folding and unfolding for the different $30 \mathrm{G}$ foldamers are most probably contained in a range covering about one order of magnitude suggests a similar distribution for the thermodynamic constant. Then the different foldamers should exhibit essentially similar thermodynamic properties. 


\section{References}

[1] Vorlickova, M.; Chladkova, J.; Kejnovska, I.; Fialova, M.; Kypr, J. Nucleic Acids Res. 2005, 33, 5851-5860.

[2] Zuker, M. Nucleic Acids Res., 2003, 31, 3406-3415. The free energies used are from the laboratory of John SantaLucia Jr (see for instance: SantaLucia, J. Jr Proc. Natl. Acad. Sci. USA, 1998, 95, 1460-1465).

[3] Tsourkas, A.; Behlke, M. A.; Rose, F. D.; Bao, G. Nucleic Acids Res., 2003, 31, 1319-1330. 